\title{
Laparoscopic resection of benign schwannoma in the hepatoduodenal ligament: A case report and review of the literature
}

\author{
LIANG TAO $^{1 *}$, SHAOYAN XU ${ }^{2 *}$, ZHIGANG REN $^{2}$, YITING LU $^{1}$, XIAOWU KONG ${ }^{1}$, \\ XIAOHUI WENG ${ }^{1}$, ZHIJIE XIE ${ }^{3}$ and ZHIMING HU ${ }^{3}$
}

\author{
${ }^{1}$ Department of General Surgery, The Third People's Hospital of Haining, Jiaxing, Zhejiang 314408; \\ ${ }^{2}$ Department of Hepatobiliary and Pancreatic Surgery, The First Affiliated Hospital, Zhejiang University School of Medicine, \\ Hangzhou, Zhejiang 310003; ${ }^{3}$ Department of Hepatobiliary and Pancreatic Surgery, Zhejiang Provincial People's Hospital, \\ Hangzhou, Zhejiang 310014, P.R. China
}

Received February 4, 2015; Accepted March 9, 2016

DOI: $10.3892 / 01.2016 .4410$

\begin{abstract}
Schwannomas are mesenchymal neoplasms with a low malignant potential, which arise from Schwann cells. The tumors can occur in most parts of the body; however, the head, neck and flexor surfaces of the extremities are the most common locations. Schwannomas occurring in the hepatoduodenal ligament are extremely rare. To the best of our knowledge, only two cases of schwannoma in the hepatoduodenal ligament have been reported in the literature, and treatment of such cases by laparoscopic surgery has not yet been reported. The present study reports a case of schwannoma in the hepatoduodenal ligament in a 50-year-old male patient. Physical and laboratory examinations showed no abnormal results. Ultrasound and computed tomography failed to definitively diagnose the mass and identify its location. During laparoscopic surgery, a mass was identified in the hepatoduodenal ligament and was completely removed. The gross specimen was a $4.5 \times 2.5 \times 2.5-\mathrm{cm}$ localized mass, yellowish-white in color. Microscopic examination revealed that the tumor was mainly composed of spindle-shaped cells and no atypical cells were identified. Immunohistochemical staining showed a strong positive S-100 protein reaction, whereas cluster of differentiation 34 and epithelial membrane antigen were negative. The final diagnosis of the lesion was benign schwannoma of the hepatoduodenal ligament. The patient was followed-up for 7 months and, at the time of writing, was healthy and without any complications. The aim of the present study was to describe a rare case of hepatoduodenal ligament schwannoma in a
\end{abstract}

Correspondence to: Dr Zhiming Hu, Department of Hepatobiliary and Pancreatic Surgery, Zhejiang Provincial People's Hospital, 158 Shangtang Road, Hangzhou, Zhejiang 310014, P.R. China E-mail: xsy200888@126.com

*Contributed equally

Key words: benign schwannoma, hepatoduodenal ligament, laparoscopic surgery, S-100 protein, computed tomography 50-year-old male patient, and present a review of the literature. To the best of our knowledge, this is the first case of hepatoduodenal ligament schwannoma treated by laparoscopic surgery.

\section{Introduction}

Schwannomas are neurogenic tumors that originate from Schwann cells, the cells of the peripheral nervous system (1). Schwannomas have been shown to occur in all ethnic groups and at all ages worldwide, with $>90 \%$ of the tumors being benign (2). Schwannomas account for $\sim 5 \%$ of benign soft-tissue neoplasms (3). Schwannomas may develop in any part of the body, but the most common sites include the head, neck and flexor surfaces of the extremities (4). Generally, schwannomas have a single place of origin, but $10 \%$ originate from multiple locations (5). Surgery is the most common treatment for schwannomas. Benign schwannomas rarely recur subsequent to complete surgical resection. Long-term survival can be expected in the majority of patients (4). Peripheral nerve sheath tumors account for 2-6\% of all gastrointestinal stromal tumors; however, schwannomas occurring in the hepatoduodenal ligament are extremely rare. To the best of our knowledge, only two such cases have been reported in the literature $(6,7)$. One patient was female and the other patient was male, aged 62 and 29 years old, respectively $(6,7)$. As schwannomas of the hepatoduodenal ligament are normally asymptomatic and often discovered incidentally, preoperative diagnosis is considerably challenging. The two patients each accepted more than two imaging examinations prior to treatment by laparotomy. The lesions were solitary and found in the hepatoduodenal ligament in the two patients. Finally, the patients were definitively diagnosed with schwannoma by pathological examination $(6,7)$. Following complete tumor excision, patients with benign schwannomas generally have a good prognosis. The present study describes the case of a hepatoduodenal ligament schwannoma in a 50-year-old male patient, and presents a review of the literature. To the best of our knowledge, this was the first time laparoscopic surgery was used for the treatment of a benign schwannoma of the hepatoduodenal ligament. 

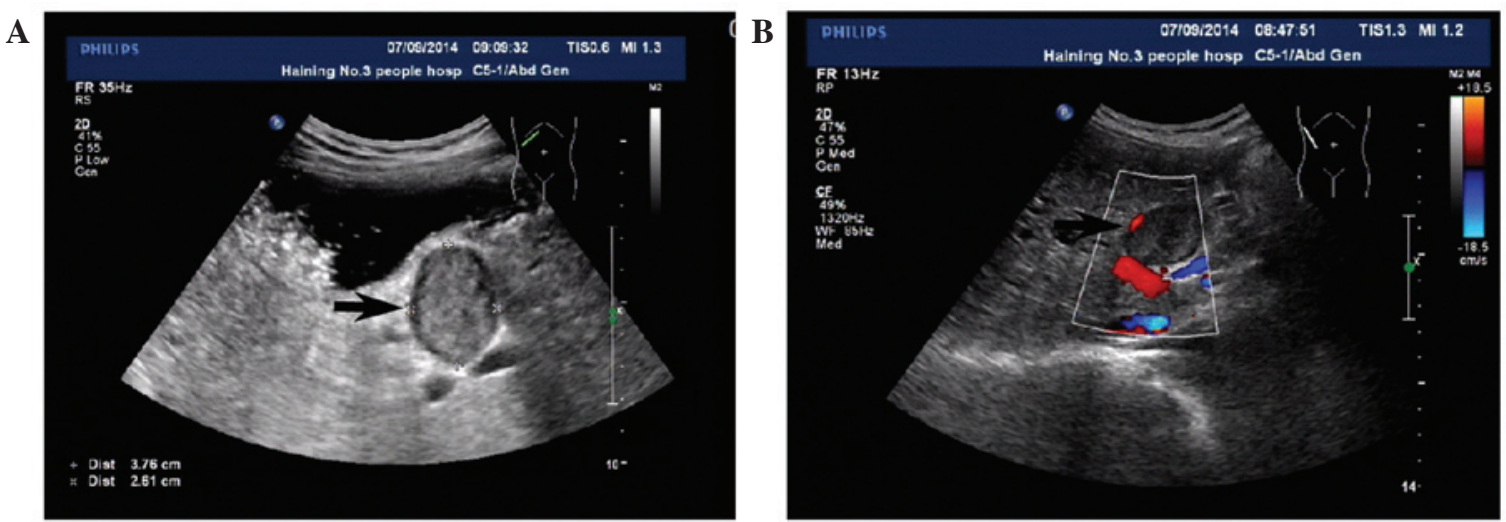

Figure 1. US and Color Doppler US. (A) Conventional US showing a mass (arrow) measuring $3.8 \times 2.6 \mathrm{~cm}$ in the space between the porta hepatis and the stomach. (B) Color Doppler US showing no blood supply in the mass (arrow). US, ultrasound.

\section{Case report}

A 50-year-old male patient was referred to the Department of General Surgery, The Third People's Hospital of Haining (Jiaxing, Zhejiang, China), presenting with pain in the right abdomen following trauma, on July 11, 2014. Physical examination revealed a soft and flat abdomen, and no enlarged lymph nodes were identified in the examinable sites. The patient's family had no history of any specific disease; however, 3 years prior to admission, a mass was incidentally detected in the hepatogastric ligament of the patient by ultrasound (US; Philips IU22; Philips, Amsterdam, Netherlands), during a health examination. As no abnormal symptoms had presented at the time, the patient did not receive any treatment. During the period of hospitalization in July 2014, an US revealed a well-defined hypodense lesion in the space between the porta hepatis and the stomach, which measured 3.6x2.4 cm (Fig. 1A). Color Doppler US (Philips IU22) showed no flow signals within the mass (Fig. 1B).

Subsequently, an abdominal computed tomography (CT) scan (GE Brightspeed Elite; Hangwei Tongyong Electric Medicine System Co., Ltd., Beijing, China) was performed to examine the region. The non-enhanced CT scan showed a well-defined round soft-tissue mass without internal calcification or liquefaction in the space between the porta hepatis and the stomach. The mass measured $3.8 \times 3.0 \times 2.5 \mathrm{~cm}$, had a uniform density and was surrounded by a clear tunica (Fig. 2A). On the contrast-enhanced CT scan, the mass showed no evident enhancement in the arterial phase (Fig. 2B). Based on the findings of the CT scan, the diagnosis of gastric stromal tumor was primarily considered. However, since the mass had been growing slowly for 3 years and possessed a clear tunica, the mass was then considered to be a benign tumor, which may be removed.

Laboratory results were as follows: Red blood cells, $4.38 \times 10^{12} / 1$ (normal values, 4.30-5.80x $10^{12} / 1$ ); white blood cells,

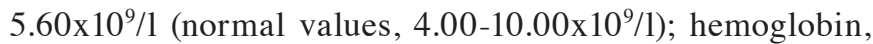
$131 \mathrm{~g} / 1$ (normal values, 130-175 g/l); lactate dehydrogenase, 146 international units (IU)/1 (normal values, 80-240 IU/1); total protein, $58.10 \mathrm{~g} / \mathrm{l}$ (normalvalues, $65.00-85.00 \mathrm{~g} / \mathrm{l}$ ); totalbilirubin, $10.80 \mu \mathrm{mol} / 1$ (normal values, 0.00-21.00 $\mu \mathrm{mol} / \mathrm{l}$ ); conjugated bilirubin, $5.70 \mu \mathrm{mol} / 1$ (normal values, $0.00-10.00 \mu \mathrm{mol} / \mathrm{l}$ ); bile acids, $2.40 \mu \mathrm{mol} / 1$ (normal values, 0.00-12.00 $\mu \mathrm{mol} / \mathrm{l}$ ); aspartate aminotransferase, $18 \mathrm{IU} / 1$ (normal values, 15-45 IU/1); alanine transaminase, 16 IU/1 (normal values, 9-60 IU/l); $\gamma$-glutamyl transpeptidase, $34 \mathrm{U} / 1$ (normal values, 10-60 IU/l); alkaline phosphatase, $67 \mathrm{U} / 1$ (normal values, 45-125 IU/1); amylase, $77 \mathrm{U} / 1$ (normal values, $<100 \mathrm{IU} / \mathrm{l}$ ); antimitochondrial antibody, negative; $\alpha$-fetoprotein, $<20 \mathrm{ng} / \mathrm{ml}$ (normal values, $<20 \mathrm{ng} / \mathrm{ml}$ ); carcinoembryonic antigen, $0.67 \mathrm{ng} / \mathrm{ml}$ (normal values, $<5.50 \mathrm{ng} / \mathrm{ml}$ ); carbohydrate antigen $19-9,4.93 \mathrm{U} / \mathrm{ml}$ (normal values, $<37.00 \mathrm{U} / \mathrm{ml}$ ); cancer antigen $125,<4.00 \mathrm{U} / \mathrm{ml}$ (normal values, $<35.00 \mathrm{U} / \mathrm{ml}$ ).

Subsequent to obtaining patient consent, laparoscopic surgery was performed (Laparoscope operating system, 22202011V110; Karl Storz GmbH \& Co. KG, Tuttlingen, Germany). The patient was placed in a horizontal position. Following the successful induction of anesthesia, the skin of the surgery field was conventionally sterilized, and sterile drapes were whisked onto the patient's body. Subsequently, a $\sim 2.0 \mathrm{~cm}$ long incision was made in the superior border of the umbilicus. $\mathrm{A} \mathrm{CO}_{2}$ pneumoperitoneum was set up with $15 \mathrm{mmHg}$ intra-abdominal pressure using a veress needle (26120J; Karl Storz GmbH \& Co. KG, Tuttlingen, Germany), and a $1.1 \mathrm{~cm}$ Trocar puncture (30103MP; Karl Storz GmbH \& Co. KG) was made to insert a laparoscopic lens (26003BA; Karl Storz GmbH \& Co. KG). Under direct vision, 3 holes were made beneath the xiphoid process and left and right upper abdomen, and the ultrasonic scalpel (GEN04; Johnson \& Johnson Medical (China) Ltd., Shanghai, China), dissecting forceps (33321ML; Karl Storz GmbH \& Co. KG), scratch-free grasping forceps (33321R; Karl Storz GmbH \& Co. KG) were inserted. During laparoscopic exploration, a mass surrounded by a fibrous capsule was located in the hepatoduodenal ligament, medial to the gallbladder and near the upper antrum (Fig. 3A and B). No invasion of the surrounding tissue was observed and the biliary ducts were not dilated. The mass was completely resected. The capsule was then removed and the gross specimen was described as a $4.5 \times 2.5 \times 2.5-\mathrm{cm}$ sized localized mass, yellowish-white in color (Fig. 3C and D). Following postoperative pathological analysis of an intraoperative frozen section, the mass was diagnosed as a schwannoma. Postoperatively, the patient had a smooth recovery and left the hospital 3 days later.

The tissue section was analyzed at the Department of Pathology, Zhejiang Provincial People's Hospital (Hangzhou, Zhejiang, China). Microscopically, the tumor mainly consisted of spindle-shaped cells, and no atypical cells or signs of malignancy were observed (Fig. 4A). Immunohistochemical 
A

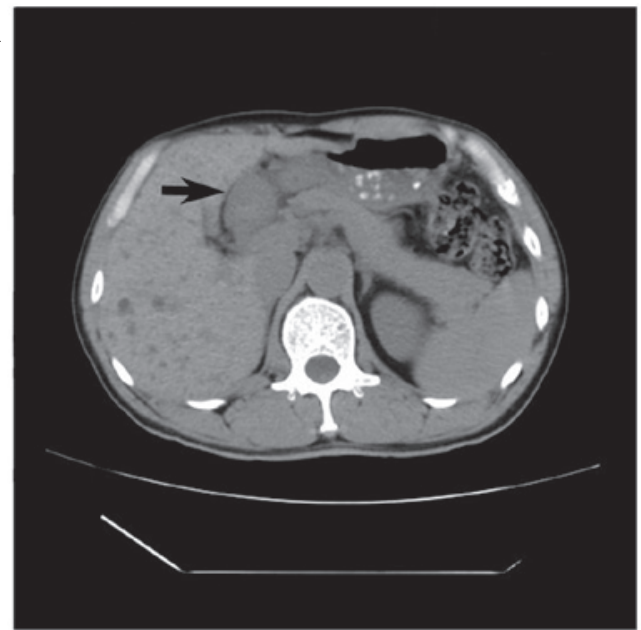

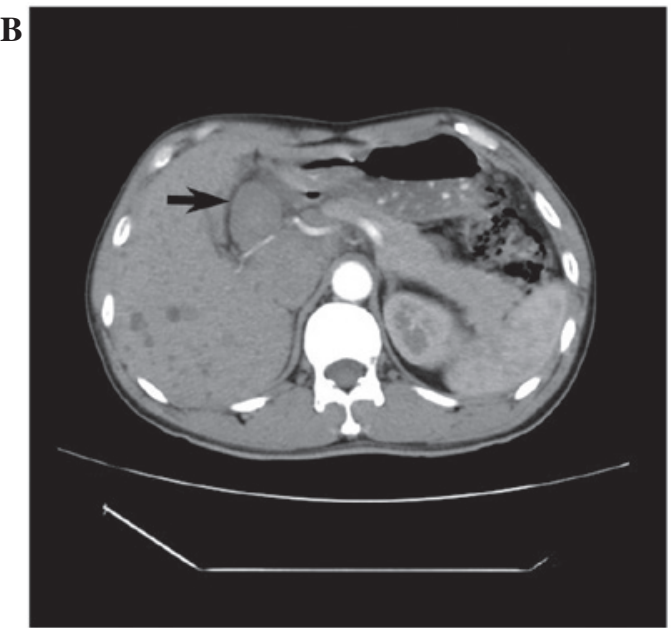

Figure 2. CT scans. (A) Non-enhanced CT scan showing a uniform density mass in the space between the porta hepatis and the stomach (arrow); (B) Enhanced CT scan showing no obvious enhancement in the arterial dominant phase (arrow). CT, computed tomography.

A

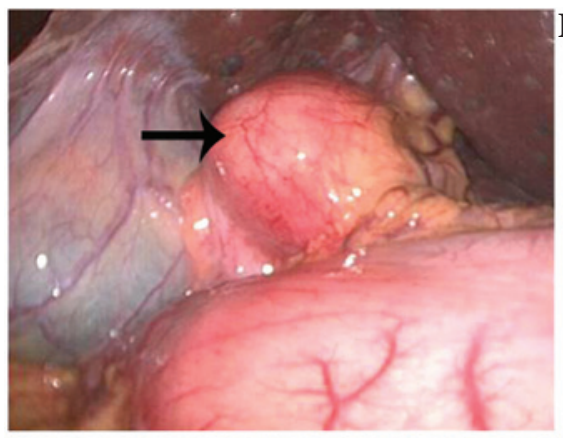

C

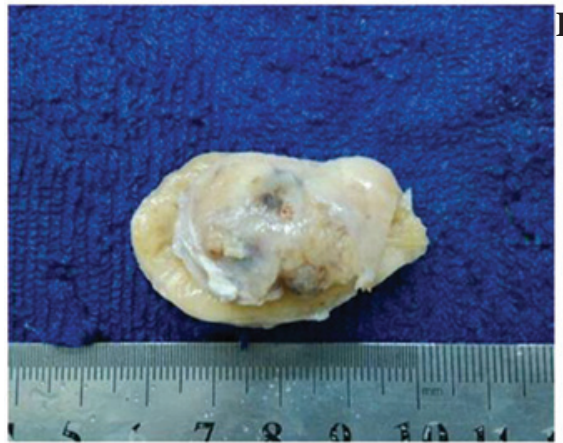

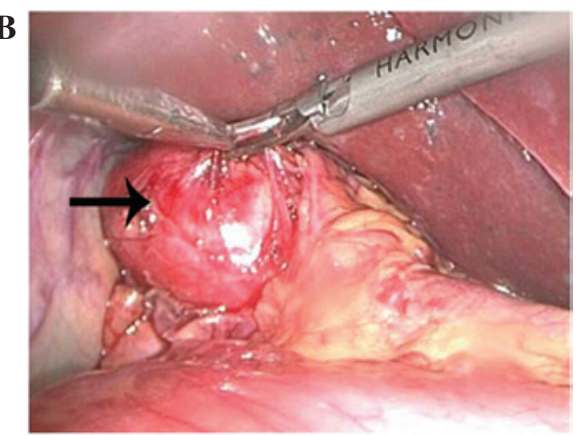

D

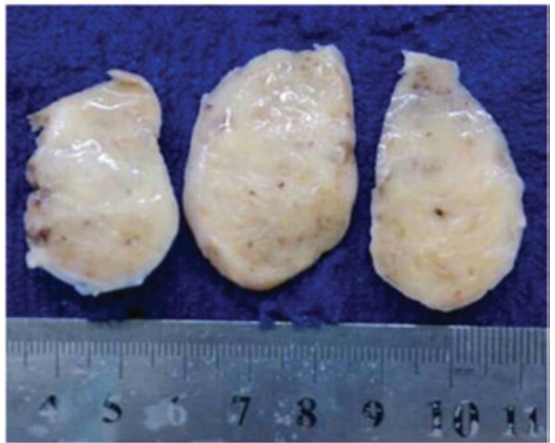

Figure 3. Intraoperative and macroscopic findings. Intraoperatively, (A) the tumor was found to be located in the hepatoduodenal ligament, (B) with no invasion of the surrounding tissue (arrows). (C) Macroscopic findings showed a capsulated yellowish-white mass. (D) The capsule was then removed and the gross specimen was described as a $4.5 \times 2.5 \times 2.5-\mathrm{cm}$ localized mass.

\section{A}

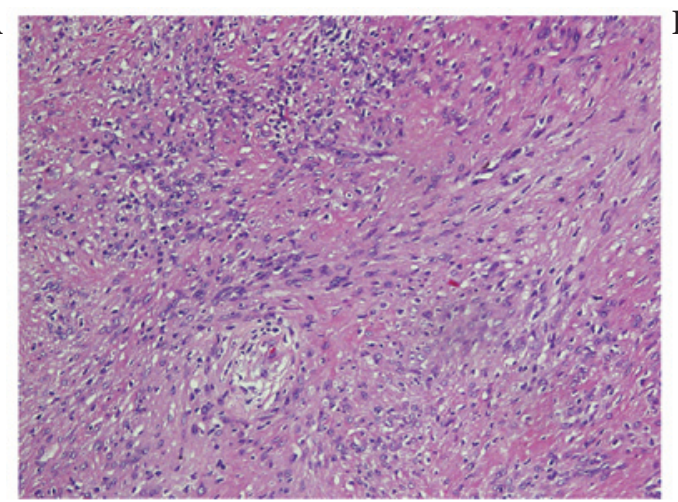

B

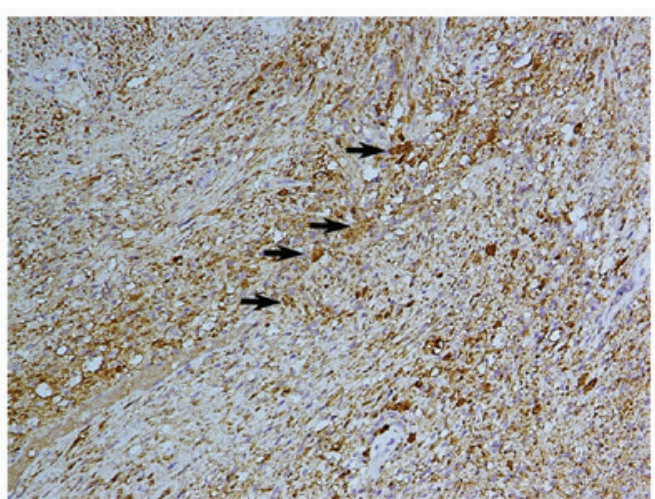

Figure 4. Pathological examination. (A) Microscopic examination showed that the tumor was mainly composed of spindle-shaped cells (stain, hematoxylin and eosin). (B) Immunohistochemical staining showed a strong tumor positivity for S-100 protein (arrows) (magnification, x10; lens diameter, 20 mm). 
analysis showed that the tumor cells were vimentin- and S-100 protein-positive (Fig. 4B), and epithelial membrane antigenand cluster of differentiation 34-negative. The final diagnosis of the tumor was schwannoma. The patient was followed-up for 7 months and, at the time of writing, was healthy and without any complications.

The present study was approved by the Ethics Committee of the Third People's Hospital of Haining (Jiaxing, Zhejiang, China).

\section{Discussion}

Schwannomas originate from the sheaths of the peripheral nerves and have been shown to occur in all ethnic groups worldwide (2). Secondary degenerative changes of schwannomas, including cyst formation, calcification, hemorrhage, and hyalinization, sometimes appear (8). Schwannomas occur in most parts of the body, but the head, neck and flexor surfaces of the extremities are the most common sites (1). They rarely occur in the retroperitoneum, accounting for $6 \%$ of all primary retroperitoneal tumors $(9,10)$. The occurrence of schwannomas in the gallbladder (11), gastroduodenal ligament (12), pancreas (13), bowel mesentery (14) or colon (15) is extremely rare. To the best of our knowledge, only two cases of schwannoma involving the hepatoduodenal ligament have been reported (6,7). The clinical characteristics of the aforementioned cases are listed in Table I. These tumors are usually asymptomatic and often incidentally discovered. Due to their low incidence and, therefore, lack of adequate understanding of the mechanism of this type of tumor, forming a preoperative diagnosis is challenging.

Prior to treatment, comprehensive imaging modalities, including US, CT and magnetic resonance imaging (MRI), should be used to establish a probable diagnosis and determine the lesion limits; however, these imaging techniques rarely provide a definitive diagnosis, due to a lack of distinguishing features of these tumors on imaging scans. Generally, schwannomas manifest as well-defined, hypoattenuating masses on non-enhanced CT scans, and on contrast-enhanced CT scans show peripheral enhancement with an irregular pattern. Delayed peripheral enhancement until the late venous phase reflects a fibrous capsule and an internal fibrillary element on CT scan (11). MRI comprises another useful tool for determining the nature of the tumor. On MRI scans, schwannomas are usually presented as masses with a low signal intensity on T1-weighted images, and inhomogeneous high signal intensity on T2-weighted images (16).

Pathology is important for a definitive diagnosis. Histologically, schwannomas consist of compact cellular lesions. Microscopically, schwannomas are encapsulated tumors with a biphasic growth pattern consisting of Antoni A (interlacing and cellular fascicles) and Antoni B (less cellular and myxoid) regions. In addition, typical Verocay bodies in the Antoni A areas may be detected using hematoxylin and eosin staining. In immunohistochemistry, positive uniform S-100 staining may be detected, which is an indicator of schwannoma $(17,18)$. In the present case, the histological findings were predominantly cellular Antoni A areas and immunohistochemistry showed a strong positive staining of the tumor cells for S-100 protein, which supported the benign nature of the schwannoma in the present case.

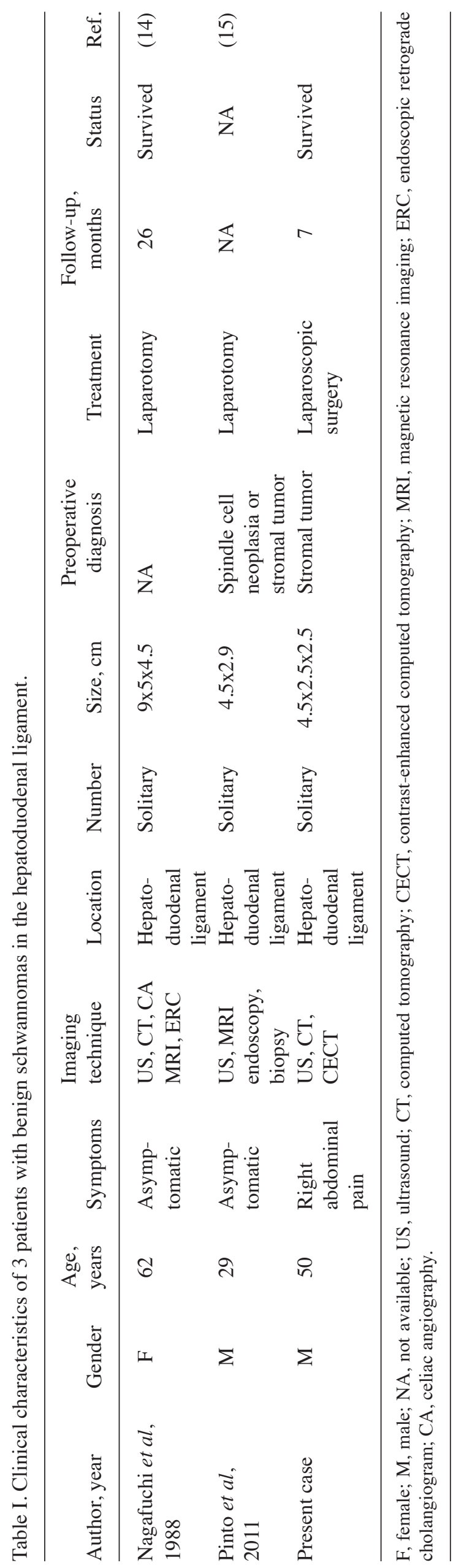


In conclusion, surgical intervention may be the optimal treatment for schwannomas, as surgery may be used to determine the location and nature of the lesion and to successfully treat it. In the present case, a large mass had developed; however, no resultant symptoms were present prior to trauma. Definitively determining the nature of the tumor preoperatively was challenging, despite the use of imaging modalities. To the best of our knowledge, this was the first case of benign schwannoma of the hepatoduodenal ligament treated by laparoscopic surgery. Following complete tumor excision, patients with benign schwannomas generally have a good prognosis.

\section{Acknowledgements}

The authors would like to thank Dr Chen Yuan (Department of Pathology, Zhejiang Provincial People's Hospital for proofreading the pathological methods.

\section{References}

1. Das Gupta TK and Brasfield RD: Tumors of peripheral nerve origin: Benign and malignant solitary schwannomas. CA Cancer J Clin 20: 228-233, 1970.

2. Ariel IM: Tumors of the peripheral nervous system. CA Cancer J Clin 33: 282-299, 1983.

3. Pilavaki M, Chourmouzi D, Kiziridou A, Skordalaki A, Zarampoukas $\mathrm{T}$ and Drevelengas A: Imaging of peripheral nerve sheath tumors with pathologic correlation: Pictorial review. Eur J Radiol 52: 229-239, 2004.

4. Le Guellec S: Nerve sheath tumours. Ann Pathol 35: 54-70, 2015 (In French).

5. Fenoglio L, Severini S, Cena P, Migliore E, Bracco C, Pomero F, Panzone S, Cavallero GB, Silvestri A, Brizio R and Borghi F: Common bile duct schwannoma: A case report and review of literature. World J Gastroenterol 13: 1275-1278, 2007.
6. Honjo Y, Kobayashi Y, Nakamura T, Takehira Y, Kitagawa M, Ikematsu Y, Ozawa T and Nakamura H: Extrahepatic biliary schwannoma. Dig Dis Sci 48: 2221-2226, 2003.

7. Lane RH, Stephens DH and Reiman HM: Primary retroperitoneal neoplasms: CT findings in 90 cases with clinical and pathologic correlation. AJR Am J Roentgenol 152: 83-89, 1989.

8. Li ZQ, Wang HY, Li J and Teng L: Recurrent retroperitoneal Schwannomas displaying different differentiation from primary tumor: Case report and literature review. World J Surg Oncol 8: 66, 2010.

9. Liu LN, Xu HX, Zheng SG, Sun LP, Guo LH and Wu J: Solitary schwannoma of the gallbladder: A case report and literature review. World J Gastroenterol 20: 6685-6690, 2014.

10. Bayraktutan U,Kantarci M, Ozgokce M, Aydinli B, Atamanalp SS and Sipal S: Education and Imaging. Gastrointestinal: Benign cystic schwannoma localized in the gastroduodenal ligament; a rare case. J Gastroenterol Hepatol 27: 985, 2012.

11. Ciledag N, Arda K and Aksoy M: Pancreatic schwannoma: A case report and review of the literature. Oncol Lett 8: 2741-2743, 2014.

12. Tang SX, Sun YH, Zhou XR and Wang J: Bowel mesentery (meso-appendix) microcystic/reticular schwannoma: Case report and literature review. World J Gastroenterol 20: 1371-1376, 2014.

13. Bugiantella W, Rondelli F, Mariani L, Peppoloni L, Cristallini E and Mariani E: Schwannoma of the colon: A case report. Oncol Lett 8: 2511-2512, 2014.

14. Nagafuchi Y, Mitsuo H, Takeda S, Ohsato K, Tsuneyoshi M and Enjoji M: Benign schwannoma in the hepatoduodenal ligament: Report of a case. Surg Today 23: 68-72, 1993.

15. Pinto J, Afonso M, Veloso R, Tente D, Fernandes S, Proença L, Carvalho J, Pontes JM and Fraga J: Benign schwannoma of the hepatoduodenal ligament. Endoscopy 43 (Suppl 2): E195-E196, 2011.

16. Rha SE, Byun JY, Jung SE, Chun HJ, Lee HG and Lee JM: Neurogenic tumors in the abdomen: Tumor types and imaging characteristics. Radiographics 23: 29-43, 2003.

17. Das Gupta TK, Brasfield RD, Strong EW and Hajdu SI: Benign solitary Schwannomas (neurilemomas). Cancer 24: 355-366, 1969.

18. Weiss SW, Langloss JM and Enzinger FM: Value of S-100 protein in the diagnosis of soft tissue tumors with particular reference to benign and malignant Schwann cell tumors. Lab Invest 49: 299-308, 1983. 